\title{
ON b-ORTHOGONALITY IN 2-NORMED SPACES
}

\author{
S. M. Gozali ${ }^{1,3}$ and H. Gunawan ${ }^{2}$ \\ ${ }^{1}$ Department of Mathematics, Institut Teknologi Bandung, Bandung, \\ Indonesia, sumanang@students.itb.ac.id \\ ${ }^{2}$ Department of Mathematics, Institut Teknologi Bandung, Bandung, \\ Indonesia, hgunawan@math.itb.ac.id
}

\begin{abstract}
In this note we discuss the concept of $b$-orthogonality in 2-normed spaces. We observe in particular that this definition of orthogonality is too loose so that every two linearly independent vectors are $b$-orthogonal.

Key words and Phrases: Orthogonality, 2-normed spaces, 2-inner product spaces.
\end{abstract}

\begin{abstract}
Abstrak. Pada makalah ini dibahas konsep ortogonalitas- $b$ di ruang norm-2. Secara khusus ditunjukkan bahwa definisi ortogonalitas- $b$ ini begitu longgar sehingga sebarang dua vektor bebas linear memenuhi ortogonalitas- $b$.
\end{abstract}

Kata kunci: Ortogonalitas, ruang norm-2, hasil kali dalam-2.

\section{Introduction}

Let $X$ be a real vector space with $\operatorname{dim}(X) \geq 2$. A real-valued function $\|\cdot, \cdot\|: X \times X \longrightarrow \mathbb{R}$ is called a 2 -norm on $X$ if the following conditions hold:

(1) $\|x, y\|=0$ if and only if $x, y$ are linearly dependent.

(2) $\|x, y\|=\|y, x\|$ for all $x, y \in X$.

(3) $\|\alpha x, y\|=|\alpha|\|x, y\|$ for all $\alpha \in \mathbb{R}, x, y \in X$.

(4) $\|x, y+z\| \leq\|x, y\|+\|x, z\|$ for all $x, y, z \in X$.

The pair $(X,\|\cdot, \cdot\|)$ is then called a 2-normed space.

${ }^{3}$ Permanent Address: Department of Mathematics Education, UPI 2000 Mathematics Subject Classification: 46B20, 46C05, 46C15, 46B99, 46C99. Received: 18-10-2010, accepted: 24-11-2010. 
For example, let $(X,\langle\cdot, \cdot\rangle)$ be an inner product space of $\operatorname{dim}(X) \geq 2$. Define the function $\|\cdot, \cdot\|_{S}$ on $X \times X$ by

$$
\|x, y\|_{S}:=\left|\begin{array}{cc}
\langle x, x\rangle & \langle x, y\rangle \\
\langle y, x\rangle & \langle y, y\rangle
\end{array}\right|^{\frac{1}{2}} .
$$

One may check that $\|\cdot, \cdot\|_{S}$ satisfies all conditions of 2-norm above. We call this the standard 2-norm on $X$.

The 2-norm concept was initially introduced by Gähler in 1960's [4]. Since then, many researchers have developed and obtained various results, see for instance $[3,5,6,7,11]$. In addition, this concept has a close relation to the so called 2-inner products (and, in general, $n$-inner products) $[2,13]$.

Geometrically, a 2-norm function generalizes the concept of area function of parallelogram due to the fact that, in the standard case, it represents the area of the usual parallelogram spanned by the two associated vectors. Observe that in a 2-normed space we have $\|x, y\|=\|x+\alpha y, y\|$ for any $\alpha \in \mathbb{R}$.

In a normed space one has different formulation for orthogonality between two vectors. At least, there are three well-known definitions of orthogonality, namely phytagorean orthogonality, isosceles orthogonality, and Birkhoff-James orthogonality [14]. In an inner product space, the three definitions are equivalent to the usual orthogonality.

Similarly in normed spaces, some researchers have studied the concept of orthogonality in 2-normed spaces. For instance, inspired by phytagorean, isosceles, and Birkhoff-James orthogonality, Khan and Siddiqui [10] defined orthogonality in 2 -normed spaces as follows. Let $(X,\|\cdot, \cdot\|)$ be a 2 -normed space, and $x, y \in X$.

(A1) $x \perp^{P} y \Leftrightarrow\|x, z\|^{2}+\|y, z\|^{2}=\|x+y, z\|^{2}$ for all $z \in X$.

(A2) $x \perp^{I} y \Leftrightarrow\|x-y, z\|=\|x+y, z\|$ for all $z \in X$.

(A3) $x \perp^{B J} y \Leftrightarrow\|x, z\| \leq\|x+\alpha y, z\|$ for all $\alpha \in \mathbb{R}, z \in X$.

See also [1] for related definition in 2-inner product spaces.

In [8], Gunawan et al. showed that this definition is 'too tight' so that one cannot find two nonzero vectors $x$ and $y$ that are orthogonal in the standard case. They revised the notion of orthogonality as follows.

(B1) $x \perp^{P} y \Leftrightarrow$ there exists a subspace $V \subseteq X$ with $\operatorname{codim}(V)=1$ such that $\|x, z\|^{2}+\|y, z\|^{2}=\|x+y, z\|^{2}$ for all $z \in V$.

(B2) $x \perp^{I} y \Leftrightarrow$ there exists a subspace $V \subseteq X$ with $\operatorname{codim}(V)=1$ such that $\|x-y, z\|=\|x+y, z\|$ for all $z \in V$.

(B3) $x \perp^{B J} y \Leftrightarrow$ there exists a subspace $V \subseteq X$ with $\operatorname{codim}(V)=1$ such that $\|x, z\| \leq\|x+\alpha y, z\|$ for all $\alpha \in \mathbb{R}, z \in V$.

Recently, inspired by Birkhoff-James orthogonality, Mazaheri et al. [9, 12] introduced the concept of $b$-orthogonality as follows. 
(C) $x \perp^{b} y \Leftrightarrow$ there exists $b \in X$ with $\|x, b\| \neq 0$ such that $\|x, b\| \leq\|x+\alpha y, b\|$ for all $\alpha \in \mathbb{R}$.

If we compare this definition to that in B3, the required condition for $b$ orthogonality is weaker. We shall see some facts concerning this concept and finally, by our investigation, we show that this definition is 'too loose'.

\section{Main Results}

Let $X=\mathbb{R}^{3}$ be equipped with the Euclidean inner product, and $\|\cdot, \cdot\|_{S}$ denote the standard 2-norm on $X$. Consider the following vectors $x=(2,0,0), y=(1,1,1)$ and $b=(1,1,0)$. By immediate evaluation, we have $\|x, b\|_{S}^{2}=4$ and $\|x+\alpha y, b\|_{S}^{2}=$ $4+2 \alpha^{2}$ for every $\alpha \in \mathbb{R}$. We conclude that $x \perp^{b} y$ but $x \not \perp y$ in the usual sense (that is, with respect to the inner product). Thus, in this space, the notion of $b$-orthogonality is not equivalent to the usual orthogonality.

The following is a characterization of $b$-orthogonality in 2-normed space in general.

Theorem 2.1. Let $X$ be any 2-normed space and $x, y \in X \backslash\{0\}$. Then, $x \perp^{b} y$ if and only if $\|x, y\| \neq 0$.

Proof. We first prove the sufficient condition. Suppose that $x, y \in X$ where $\|x, y\| \neq 0$ or equivalently, $y \neq k x$ for any $k \in \mathbb{R}$. By choosing $b=y$, we have $\|x+\alpha y, b\|=\|x, b\|$ for all $\alpha \in \mathbb{R}$. Thus we find that $x \perp^{b} y$.

To prove the necessary condition, take $x \in X$ and $y=k x$ for some $k \in \mathbb{R} \backslash\{0\}$. Then, for any $b \in X$ with $\|x, b\| \neq 0$, we have $\|x+\alpha y, b\|=|1+k \alpha|\|x, b\|<\|x, b\|$ for some $\alpha \in\left(-\frac{2}{|k|}, \frac{2}{|k|}\right)$. Therefore, $x \not \not^{b} y$.

Based on this theorem, we observe that any two linearly independent vectors $x, y$ in 2-normed space of any dimension satisfy $x \perp^{b} y$ and $y \perp^{b} x$. In this regard, we see that the definition of $b$-orthogonality is too loose.

Let us now try to fix the situation by tightening slightly the condition for the orthogonality. Suppose that for $x$ and $y$ to be $b$-orthogonal, we require that there must exist $b \in X$ with $\|x, b\| \neq 0$ and $\|y, b\| \neq 0$ such that $\|x, b\| \leq\|x+\alpha y, b\|$ for all $\alpha \in \mathbb{R}$. We see that with this new condition, the necessary part of Theorem 1 still holds. What remains is to check the sufficient part, that is, if $\|x, y\| \neq 0$ implies $x \perp^{b} y$. 
In the two-dimensional case, we have the following observation. Suppose that $b=c x+d y$ for some nonzero scalars $c$ and $d$. Then, we have

$$
\begin{aligned}
\|x+\alpha y, b\| & =\|x+\alpha y, c x+d y\| \\
& =\|x+\alpha y, x+(d / c) y\||c| \\
& =\|x+\alpha y, x+\alpha y+(d / c-\alpha) y\||c| \\
& =\|x+\alpha y,(d-\alpha c) y\| \\
& =\|x,(d-\alpha c) y\|, \quad \text { whenever } \alpha \neq d / c .
\end{aligned}
$$

Now we always can find $\alpha$ such that $\alpha \neq d / c$ and $\|x,(d-\alpha c) y\|<\|x, b\|=\|x, d y\|$. This tells us that in a two-dimensional space, any two vectors are not $b$-orthogonal.

Let us now turn to the space of dimension $d \geq 3$. Here we may try to look for $b \notin \operatorname{span}\{x, y\}$ which satisfies the above condition. Our next observation shows that the new definition is still too loose, for in the standard 2-normed space we find that any two linear independent vectors are $b$-orthogonal.

To see this, let $(X,\langle\cdot, \cdot\rangle)$ be an inner product space of dimension $d \geq 3$. We define the standard 2-inner product on $X$ by

$$
\langle x, y \mid z\rangle:=\left|\begin{array}{cc}
\langle x, y\rangle & \langle x, z\rangle \\
\langle z, y\rangle & \langle z, z\rangle
\end{array}\right|
$$

Note that $\|x, z\|_{S}:=\langle x, x \mid z\rangle^{\frac{1}{2}}$ is the standard 2-norm discussed earlier. In connection with $b$-orthogonality, we have the following theorem.

Theorem 2.2. Let $x, y \in X$ be fixed. For any $b \in X$ we have

$$
\|x, b\|_{S} \leq\|x+\alpha y, b\|_{S} \text { for all } \alpha \in \mathbb{R} \Leftrightarrow\langle x, y \mid b\rangle=0 .
$$

Proof. $(\Leftarrow)$ If $\langle x, y \mid b\rangle=0$, then

$$
\begin{aligned}
\|x+\alpha y, b\|_{S}^{2} & =\langle x+\alpha y, x+\alpha y \mid b\rangle \\
& =\|x, b\|_{S}^{2}+2 \alpha\langle x, y \mid b\rangle+\alpha^{2}\|y, b\|_{S}^{2} \\
& \geq\|x, b\|_{S}^{2} .
\end{aligned}
$$

for all $\alpha \in \mathbb{R}$.

$$
(\Rightarrow) \text { Suppose that }\|x, b\|_{S} \leq\|x+\alpha y, b\|_{S} \text { for all } \alpha \in \mathbb{R} \text {. Then }
$$

$$
2 \alpha\langle x, y \mid b\rangle+\alpha^{2}\|y, b\|_{S}^{2} \geq 0
$$

for all $\alpha \in \mathbb{R}$. This is true only when $\langle x, y \mid b\rangle=0$. (For otherwise, there will be some value of $\alpha$ for which $2 \alpha\langle x, y \mid b\rangle+\alpha^{2}\|y, b\|_{S}^{2}<0$.)

This fact suggests to us that obtaining $b$ satisfying $\langle x, y \mid b\rangle=0$ is equivalent to having $x \perp^{b} y$. Our next theorem is the following.

Theorem 2.3. Let $X$ be equipped with standard 2-norm. If $\|x, y\| \neq 0$ then there exists $b \in X$ such that $\langle x, y \mid b\rangle=0$. 
Proof. Suppose that $x, y \in X$ where $\|x, y\| \neq 0$ or equivalently, $y \neq k x$ for any $k \in \mathbb{R}$. If $\langle x, y\rangle=0$, we only need to take $b$ such that $\langle x, b\rangle=0$ or $\langle y, b\rangle=0$ to obtain $\langle x, y \mid b\rangle=0$.

Suppose now that $\langle x, y\rangle \neq 0$. Using the fact that $\langle x, y \mid b\rangle=0$ implies $\langle\lambda x, \mu y \mid b\rangle=0$ for any $\lambda, \mu \in \mathbb{R}$, we may assume that $\|x\|=\|y\|=1$. Now suppose that $z \perp \operatorname{span}\{x, y\}$ and $\|z\|=1$, and consider $b:=x \pm y+\beta z$. Then, we have

$$
\|b\|^{2}=2 \pm 2\langle x, y\rangle+\beta^{2} .
$$

By the definition of the standard 2-inner product, one may observe that

$$
\langle x, y \mid b\rangle=\langle x, y\rangle\|b\|^{2}-\langle x, b\rangle\langle b, y\rangle=0 \Leftrightarrow \beta^{2}= \pm \frac{1-\langle x, y\rangle^{2}}{\langle x, y\rangle} .
$$

We choose the sign to be the same as the sign of $\langle x, y\rangle$, so that $\pm\langle x, y\rangle$ is definite positive. Therefore, we can choose the value of $\beta$ from the above equation to obtain $b$ for which $\langle x, y \mid b\rangle=0$.

\section{Concluding Remarks}

Our results show that the notion of $b$-orthogonality introduced by Mazaheri and used in his other papers is too loose, in the sense that talking about two $b$-orthogonal vectors amounts to talking about two linearly independent vectors. We further show that even if we add a requirement that the vector $b$ must also be linearly independent on $y$, the situation is the same in the standard 2-normed space of dimension $d \geq 3$.

With our results, one should therefore rethink: what is the use of $b$-orthogonality if it is nothing more than asking whether two vectors are linearly independent or not. The use of the existential quantor in the condition is clearly inappropriate. As an alternative, one should use the universal quantor, as in [8]. In fact, Gunawan et al. have shown that their definition of orthogonality in 2-normed spaces, when restricted to the standard case, is equivalent to the usual orthogonality (with respect to the given inner product).

Acknowledgement. This work is supported by ITB Research Grant No. 221/2010.

\section{References}

[1] Cho, Y. J. and Kim, S. S., "Gateaux Derivatives and 2-Inner Product Spaces" Glas. Mat. Ser. III 27 (1983), 197-203.

[2] Diminnie, C. R. and White, A., "A Characterization of 2-Inner Product Spaces", Math. Japon. 114 (1983), 275-277. 
[3] Dragomir, S. S., Cho, Y. J. and Kim, S. S., "Superadditivity and Monotonicity of 2-Norms Generated by Inner Products and Related Results", Soochow J. Math. 24 (1998), 13-32.

[4] Gähler, S., "Lineare 2-Normierte Räume", Math. Nachr. 28 (1964), 1-43.

[5] Gunawan, H., "On $n$-Inner Products, $n$-Norms, and the Cauchy-Schwarz Inequality", Sci. Math. Jpn. 55 (2002), 53-60.

[6] Gunawan, H., "Inner Product on n-Inner Product Spaces", Soochow J. Math. 28 (2002), 389-398.

[7] Gunawan, H. and Mashadi, "On n-Normed Spaces", Int. J. Math. Math. Sci. 27 (2001), 631-639.

[8] Gunawan, H., Mashadi, Gemawati, S., Nursupiamin and Sihwaningrum, I., "Orthogonality in 2-Normed Spaces Revisited", Univ. Beograd Publ. Elektrotehn. Fak. Ser. Mat. 17 (2006), $76-83$.

[9] Kamali, H.R. and Mazaheri, H., "b-General Orthogonality in 2-Normed Spaces", Int. Appl. Math. Sci. 2 (2008), 775-780.

[10] Khan, A. and Siddiqui, A., "B-Orthogonality in 2-Normed Space", Bull. Calcutta Math. Soc. 74 (1982), 216-222.

[11] Mazaheri, H. and Kazemi, R., "Some Results on 2-Inner Product Spaces", Novi Sad J. Math. 37 (2007), 35-40.

[12] Mazaheri, H. and Nezhad, S. G., "Some Results on b-Orthogonality in 2-Normed Linear Spaces", Int. J. Math. Anal. 1 (2007), 681-687.

[13] Misiak, A., "n-Inner Product Spaces", Math. Nachr. 140 (1989), 299-319.

[14] Partington, J. R., "Orthogonality in Normed Spaces", Bull. Austral. Math. Soc. 33 (1986), 449-455. 